Full Length Article

\title{
Laser induced periodic surface structures formation by nanosecond laser irradiation of poly (ethylene terephthalate) reinforced with Expanded Graphite
}

\author{
René I. Rodríguez-Beltrán ${ }^{\mathrm{a}, \mathrm{b}, *}$, Margarita Hernandez ${ }^{\mathrm{b}}$, Sandra Paszkiewicz ${ }^{\mathrm{c}}$, \\ Anna Szymczyk ${ }^{\mathrm{c}}$, Zbigniew Rosłaniec ${ }^{\mathrm{c}}$, Tiberio A. Ezquerra ${ }^{\mathrm{d}}$, Marta Castillejo ${ }^{\mathrm{b}}$, \\ Pablo Moreno ${ }^{\mathrm{a}}$, Esther Rebollar ${ }^{\mathrm{b}, *}$ \\ ${ }^{a}$ Grupo de Aplicaciones del Láser y Fotónica (ALF-USAL), Universidad de Salamanca, Pl. de la Merced s/n, 37008 Salamanca, Spain \\ b Instituto de Química Física Rocasolano (IQFR-CSIC), C/Serrano 119, 28006 Madrid, Spain \\ ${ }^{c}$ West Pomeranian University of Technology, Piastow Av. 19, PL-70310 Szczecin, Poland \\ ${ }^{\mathrm{d}}$ Instituto de Estructura de la Materia (IEM-CSIC), C/Serrano 121, 28006 Madrid, Spain
}

\section{A R T I C L E I N F O}

\section{Article history:}

Received 29 September 2017

Received in revised form

14 December 2017

Accepted 18 December 2017

Available online 18 December 2017

\section{Keywords:}

Expanded graphite

Nanocomposite

Laser induced periodic surface structures

Surface free energy

Adhesion

\begin{abstract}
A B S T R A C T
We report on the formation of Laser Induced Periodic Surface Structures in poly (ethylene terephthalate) and poly (ethylene terephthalate)/Expanded Graphite films by laser irradiation with nanosecond pulses at $266 \mathrm{~nm}$. The characterization studies show that the quality of the ripples depends strongly on the irradiation time and fluence and the optimal conditions for obtaining LIPSS are affected by the amount of the expanded graphite present in the film due to the differences in crystallinity, thermal conductivity and thermal diffusivity of the nanocomposites. Physicochemical modifications in the materials were inspected by Raman spectroscopy, the colloidal probe technique and contact angle measurements using different liquids. Results show that there is an increase of the hydrophilicity of the surfaces after laser irradiation together with an increase of the surface free energy and in particular of its polar component. Additionally, the adhesion force estimated by the colloidal probe technique increases after laser nanostructuring.
\end{abstract}

(C) 2017 Elsevier B.V. All rights reserved.

\section{Introduction}

Polymer-based nanocomposites reinforced with carbon nanomaterials such as graphite [1,2], graphene [3,4], or carbon nanotubes [5,6], have shown significant improvement in their mechanical properties [7,8], their electrical [7,9] and thermal $[10,11]$ conductivities, and barrier impermeability to vapours and gases [12], in comparison to the unmodified polymers.

Expanded Graphite (EG) is a material derived from natural graphite [13]. It has a layered/planar structure, where the atoms are covalently bonded in a honeycomb lattice in each layer, while bonding is weak perpendicularly to the plane. The synthesis of EG is well documented in the literature [13]. It is synthetized by means of a two-step process. Natural graphite is first converted into inter-

\footnotetext{
* Corresponding authors at: Instituto de Química Física Rocasolano (IQFR-CSIC), C/Serrano 119, 28006 Madrid, Spain.

E-mail addresses: renrobe@usal.es (R.I. Rodríguez-Beltrán), e.rebollar@csic.es (E. Rebollar).
}

calated or expandable graphite through chemical oxidation in the presence of concentrated $\mathrm{H}_{2} \mathrm{SO}_{4}$ and $\mathrm{HNO}_{3}$ acids. Then, a reduction process which consists in exposing the expandable compound to high temperatures is performed in a furnace above $600^{\circ} \mathrm{C}$. These high temperatures produce a rapid expansion and the exfoliation of graphite. The obtained EG can be further chemically modified to enhance affinity of carbon material with a polymer matrix [14,15].

Different polymers have been reinforced with EG showing an improvement of their physical properties. For instance, improvement in both electrical conductivity and structural integrity was observed in poly (methyl methacrylate) (PMMA) [16], thermal, mechanical, and electrical properties are improved significantly in polystyrene (PS) [17], and mechanical properties and electrical conductivity were enhanced in poly (ethylene terephthalate)(PET)[18] and both electrical and barrier properties were improved along with the incorporation of EG in poly(trimethylene terephthalate) [19].

In addition to the modification of the material properties by the use of additives, a different way of providing a certain functionality consists in the patterning of polymer surfaces in the micro- 
and nanoscale. Micro- and nanostructures have been reported to modify the optical properties of the polymer surface by providing antireflective properties [20,21] or reflective ones, which allows the use as photonic crystals [22], to modify the hydrophobic or hydrophilic character [21,23], or to improve light harvesting for more efficient organic solar cells [24]. In particular, surface structuring of carbon-based polymer composites by nanoimprint lithography (NIL) has been reported to modify the wettability and mechanical properties of the materials [25] and demonstrated that the fabrication of materials with different functionalities is possible by combining the use of fillers and the control of the surface structure. Although lithographic methods are commonly used for generating polymer structures at the nanoscale [26-28] and aim at reproducibility and low cost, they require multiple-step procedures involving clean-room facilities, high vacuum or complex mask fabrication. As an alternative, laser surface patterning techniques such as the formation of Laser Induced Periodic Surface Structures (LIPSS) have demonstrated to provide versatility and reliability and constitute potential methods to obtain large processed surface areas in many types of materials [29-32], including polymers [33-35]. The formation of LIPSS in polymer surfaces has been observed by using lasers with pulse duration from nanosecond (ns) to femtosecond (fs), at wavelengths from the ultraviolet to the infrared [33,34,36-38]. In a ns-pulse irradiation scenario, this technique requires the irradiation of the surface of the sample with linearly polarized laser pulses, at a wavelength efficiently absorbed by the polymer and fluences well below the ablation threshold $[33,36]$. The formation of LIPSS can be explained as the result of the interference between the incident and the surface-scattered waves, and a positive feedback process $[33,36,39]$. During irradiation, the polymer material is heated and the temperature for amorphous and semicrystalline polymers must overcome the glass transition temperature $\left(T_{g}\right)$ or the melting temperature $\left(T_{m}\right)$, respectively, in order to allow the rearrangement of the polymer chains. This leads to the formation of structures with a spatial period $(L)$ close to the laser wavelength $(\lambda)$ and aligned parallel to the polarization of the laser beam. Such spatial period is strongly dependent of the angle of incidence $(\theta)$ of the laser beam and of the effective refraction index $\left(n_{e}\right)$ of the material. So, it can be calculated according to the following expression [40]:

$L=\frac{\lambda}{n_{e}-\sin (\theta)}$

Previously, some of us have reported the formation of LIPSS on thin films of PET by irradiating with both ns [36] and fs laser pulses $[37,41]$. In this work, we present a systematic study of the formation of ripple-like nanostructures in free-standing PET films reinforced with EG using UV ns pulsed laser irradiation. Additionally, we report on the influence of the additive content on the LIPSS formation, and on the modification of surface properties, such as wetting behavior and adhesion forces, which are relevant for the potential future applications. For this purpose, measurements of atomic force microscopy (AFM) have been performed to analyse the morphology of the LIPSS. Raman spectroscopy was carried out in order to identify possible chemical modifications during LIPSS formation. Also, AFM employing a colloidal probe was used to characterize the adhesion of non-irradiated and irradiated samples and contact angle (CA) characterization was achieved with different liquids in order to determine the surface free energy components in the polymer and in the nanocomposite, before and after irradiation.

\section{Experimental}

In this work, films of PET and EG reinforced PET were used. These samples were prepared by the in situ polymerization method reported by Paszkiewicz, et al. [42]. Regarding the PET/EG samples,
EG concentrations of $0.025,0.05,0.1,0.2$ and $0.4 \mathrm{wt}$. \% were used for this study. It was previously reported that these nanocomposites exhibit electrical conductivity with a low percolation threshold of $\approx 0.05 \mathrm{wt} . \%$ and a high electrical conductivity of $10^{-3} \mathrm{~S} / \mathrm{cm}$ was achieved with only 0.4 wt.\% of exfoliated EG [42].

PET and its composites were melted at $260^{\circ} \mathrm{C}$ and pressed for 2 min under a pressure of 5 bar and 2 min under 10 bar. Films with a thickness of approximately $0.4 \mathrm{~mm}$ and an average roughness $\left(\mathrm{R}_{\mathrm{a}}\right)$, as revealed by AFM, of $3.9 \pm 0.1$ and $4.7 \pm 0.3 \mathrm{~nm}$ for PET and PET/EG 0.4 wt.\% respectively, were obtained. Wide angle X-ray scattering (WAXS) measurements were carried out using a Panalytical XPert PRO diffractometer ( $\mathrm{Cu} \mathrm{K}$-alpha $\lambda=0.154 \mathrm{~nm}$ ) in order to determine the crystallinity of the samples after the preparation process.

Before irradiation, the polymer and composites were deposited in quartz windows by spin coating in order to measure the UV-Vis spectrum. The equipment used for this purpose was a UV-Visible spectrometer (UV-VIS-3600 Shimadzu). The value of the absorption coefficient, at $266 \mathrm{~nm}$, was estimated to be $2 \pm 0.2 \times 10^{4} \mathrm{~cm}^{-1}$ for PET and $1.8 \pm 0.1 \times 10^{4} \mathrm{~cm}^{-1}$ for PET/EG $0.4 \mathrm{wt}$.\% films. Laser irradiation was carried out using a linearly polarized laser beam from a Q-Switched Nd:YAG system (Lotis TII LS-2131M), at a wavelength corresponding to the fourth harmonic of the fundamental laser emission $(266 \mathrm{~nm})$, a pulse duration of $8 \mathrm{~ns}$ and a repetition rate of $10 \mathrm{~Hz}$. Irradiation experiments were performed in ambient air, at normal incidence and varying the number of pulses and the fluence. The fluences used were well below the fluence ablation threshold for a single pulse, which was determined to be $82 \pm 11 \mathrm{~mJ} / \mathrm{cm}^{2}$ in both cases.

Topography was inspected by atomic force microscopy (AFM) using a Multimode 8 AFM equipped with a Nanoscope $V$ controller by Bruker in tapping mode with silicon probes (NSG30 by NTMDT). AFM images were analyzed using the software Nanoscope Analysis 1.50 (Bruker) in order to obtain statistical data about the period and height (distance from peak to valley) of LIPSS. Additionally, adhesion measurements were carried out in AFM by the colloidal probe technique [43] in order to characterize the surface forces in the micrometer range. A colloidal probe (CPFM_SiO2-A/Au by NT-MDT) makes contact with the surface and then is retracted in a single measurement while monitoring both approaching and retracting force-distance curves. Three sets of curves per sample were recorded in order to achieve statistically significant results. The cantilever spring constant was measured by the thermal tune method and found to be around $1.6 \mathrm{~N} / \mathrm{m}$.

Contact angle measurements were performed in irradiated and non-irradiated materials, using a pocket goniometer PG2 (FIBRO system). The static wetting CA was measured at room temperature and ambient humidity with deionized water, paraffin oil and glycerol (both of them supplied by Sigma-Aldrich) as liquid probes. A statistical analysis to obtain each value of CA was carried out, performing eight measurements per sample. The surface free energy components were calculated from the measured CA values.

Raman spectroscopy was used in order to detect possible chemical changes in samples after irradiation. The spectra were recorded using a micro-Raman spectrometer (Renishaw InVia 0310-02), equipped with a laser excitation source operating at a wavelength of $785 \mathrm{~nm}$ and a CCD camera. The spectra were acquired with a spectral resolution of $2 \mathrm{~cm}^{-1}$ using a $50 \times$ magnification objective to a spot with a diameter $<1 \mu \mathrm{m}$ on the samples. Laser power conditions were those that ensured the integrity of the polymer.

\section{Results}

LIPSS were obtained for both PET and its EG nanocomposites. As an example, Fig. 1 displays AFM topography images of PET (upper row) and PET/EG 0.4 wt.\% (bottom row) samples before irradia- 

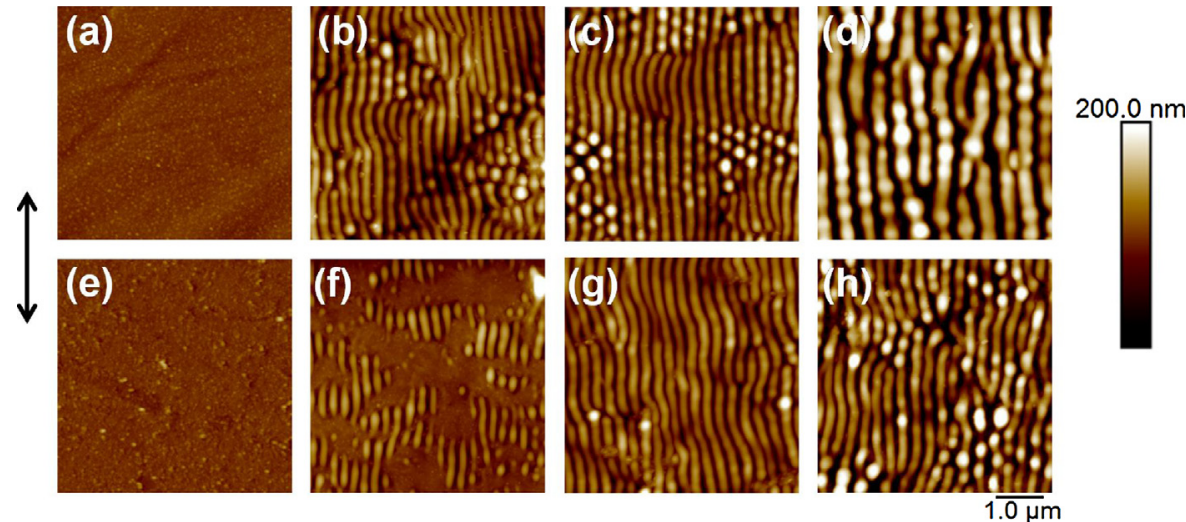

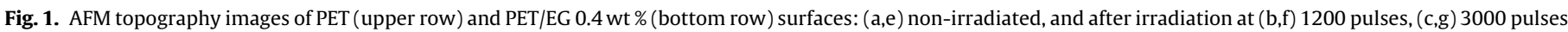
and $(\mathrm{d}, \mathrm{h}) 4800$ pulses. Irradiation was performed at $\lambda=266 \mathrm{~nm}$ and a laser fluence of $8 \mathrm{~mJ} / \mathrm{cm}^{2}$. The arrow indicates the direction of the polarization of the laser beam.

tion, and the evolution of the formation of LIPSS generated by laser irradiation with different number of pulses and a fixed fluence of $8 \mathrm{~mJ} / \mathrm{cm}^{2}$. In both cases, ripples obtained have a period close to the irradiation wavelength and they are parallel to the polarization of the laser beam. It is worth remarking that LIPSS are formed without ablation of the material, as can be observed in Fig. S1.

The results of the statistical study of both periods and heights are plotted in Fig. 2. In the case of PET, the formation of LIPSS starts after 200 pulses. The period increases with the number of pulses up to 2400 pulses and it remains constant afterwards close to the value of the laser wavelength. The height of the ripples rises abruptly after 300 pulses from around $30 \mathrm{~nm}$ to $75 \mathrm{~nm}$ (at 600 pulses) and then it remains constant. For a number of pulses over 3600 , the structures begin to deteriorate. In the same way, the evolution of LIPSS as a function of the laser fluence for a fixed number of pulses was studied. It can be observed in Fig. $2 \mathrm{~b}$, that the formation of LIPSS takes place within the range of $6-8 \mathrm{~mJ} / \mathrm{cm}^{2}$. Fig. $2 \mathrm{~b}$ also shows that the height and period of the ripples can be considered constant in the narrow range of fluences that allows LIPSS formation. The period is close to the laser wavelength, while the structures have a height between 80 and $90 \mathrm{~nm}$.

The dependence of LIPSS formation with both the number of pulses and the laser fluence was also studied for the PET/EG 0.4 wt.\% composite and the results are shown in Fig. 2. In this case, a higher amount of pulses is needed in order to observe LIPSS. Regarding the dependence with the number of pulses, the period remains constant up to 2400 pulses and then it slightly increases for a higher number of pulses, reaching a maximum value for 3600 pulses and it remains constant afterwards. Regarding the dependence on fluence, the range of fluence for the formation of LIPSS in PET/EG
0.4 wt.\% is broader than that for PET. It starts at $6 \mathrm{~mJ} / \mathrm{cm}^{2}$ and from $10 \mathrm{~mJ} / \mathrm{cm}^{2}$ on, the structures begin to deteriorate.

As shown in Fig. 3a, while PET films were essentially amorphous, the composite exhibited significant traces of crystallinity. This is related to the presence of EG which acts as a nucleating agent, as previously described [42]. PET/EG samples with different EG concentrations were irradiated by using a fluence of $8 \mathrm{~mJ} / \mathrm{cm}^{2}$ and 3000 pulses, in order to compare the values of period and height with those of PET and PET/EG 0.4 wt.\%. AFM images are shown in Figure S2. The statistical study is shown in Fig. $3 \mathrm{~b}$. The values of period and height decrease as the concentration of EG in the polymer matrix increases.

Samples nanostructured upon irradiation with 3000 pulses and $8 \mathrm{~mJ} / \mathrm{cm}^{2}$ were further characterized in order to assess possible physicochemical modifications which take place simultaneously to LIPSS formation.

Fig. 4 shows the Raman spectra corresponding to both nonirradiated and irradiated samples of PET and PET/EG 0.4 wt.\%. The Raman bands in PET samples were ascribed to molecular bond vibrations, according to the assignments of reference [44]. In the case of PET/EG $0.4 \mathrm{wt} . \%$, a difference is observed in the relative intensity of the bands at 1095 and $1117 \mathrm{~cm}^{-1}$, which can be ascribed to the different crystalline content. Highly crystalline samples exhibit a well-defined band at $1095 \mathrm{~cm}^{-1}$, whereas amorphous samples show a shoulder at that wavenumber on the $1117 \mathrm{~cm}^{-1}$ band [45]. Additionally, a new band appears located at $1582 \mathrm{~cm}^{-1}$, assigned to the G-band of graphite. No significant differences are observed between the spectra corresponding to the non-irradiated and irradiated samples, neither in the case of PET nor for the PET/EG $0.4 \mathrm{wt} . \%$.

(b)

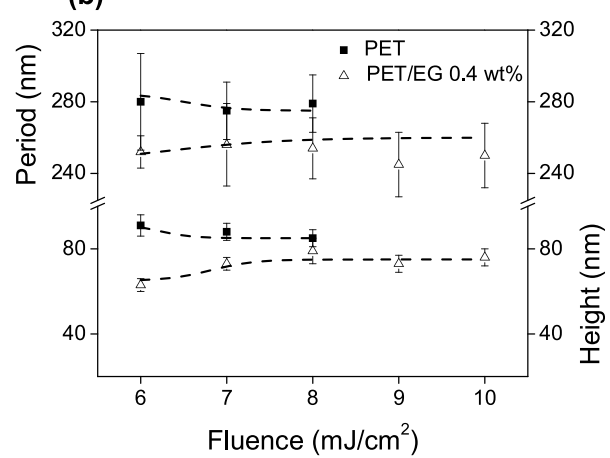

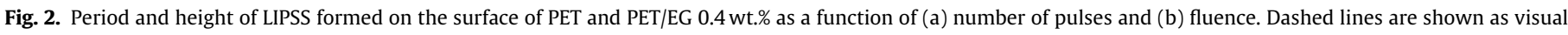
guides. 
Table 1

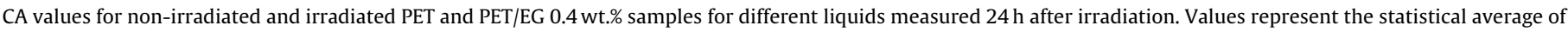
eight measurements.

\begin{tabular}{|c|c|c|c|}
\hline \multirow[b]{2}{*}{ Sample } & \multicolumn{3}{|c|}{ Contact angle $\left(^{\circ}\right)$} \\
\hline & Water & Glycerol & Paraffin oil \\
\hline PET, non-irradiated & $77 \pm 3$ & $64 \pm 2$ & $20 \pm 2$ \\
\hline PET, irradiated & $52 \pm 4$ & $64 \pm 2$ & $27 \pm 1$ \\
\hline PET/EG 0.4 wt.\%, non-irradiated & $76 \pm 2$ & $65 \pm 1$ & $23 \pm 3$ \\
\hline PET/EG 0.4 wt.\%, irradiated & $51 \pm 3$ & $67 \pm 3$ & $30 \pm 2$ \\
\hline
\end{tabular}

(a)

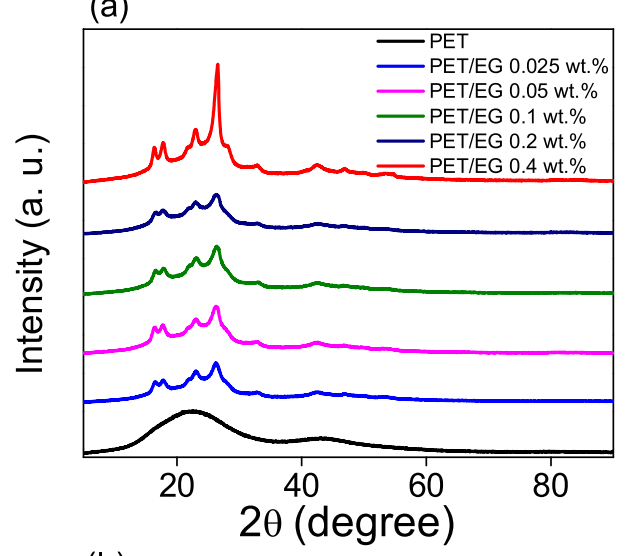

(b)

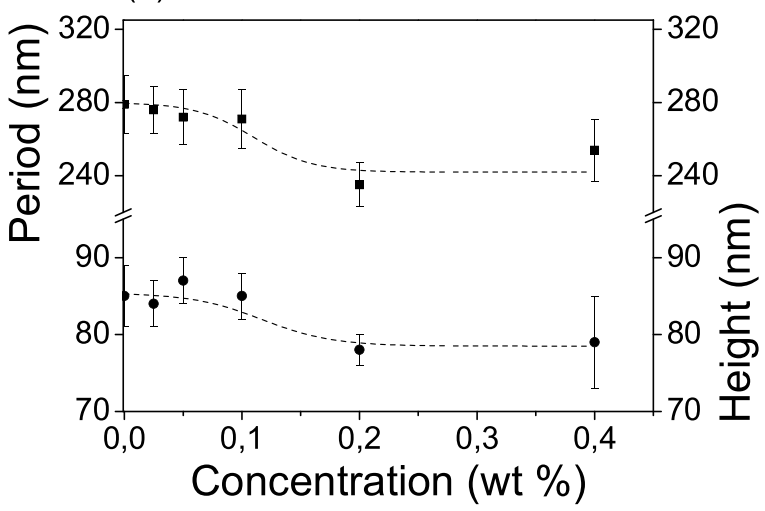

Fig. 3. (a) Diffractograms for PET and PET/EG with different EG concentrations (b) Period and height of LIPSS formed in PET and PET/EG samples with different EG concentrations irradiated by using a fluence of $8 \mathrm{~mJ} / \mathrm{cm}^{2}$ and 3000 pulses.

To further analyse the possible physicochemical surface modifications induced by laser irradiation, CA of water, glycerol and paraffin oil were measured on the surface of the non-irradiated and irradiated samples. As an example, Fig. 5 shows water, glycerol and paraffin oil droplets on non-irradiated and irradiated PET/EG $0.4 \mathrm{wt} . \%$ surfaces $24 \mathrm{~h}$ after irradiation. Additional analysis was carried out immediately after irradiation and $96 \mathrm{~h}$ after irradiation and

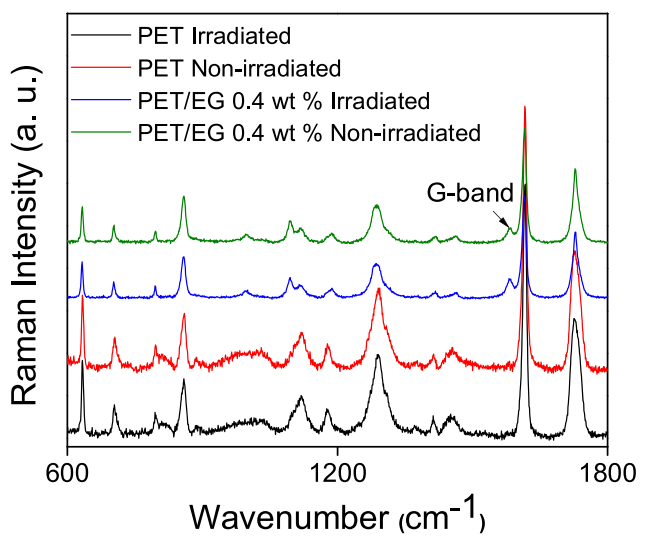

Fig. 4. Raman spectra for non-irradiated and irradiated PET and PET/EG 0.4 wt.\% surfaces.

it was observed that CA values remain constant in time. The CA value, shown in red, is an average of eight individual measurements. The results for non-irradiated and irradiated samples for each liquid probe are shown in Table 1.

Both PET and PET/EG 0.4 wt.\% are slightly hydrophilic as shown by the water CA smaller than $90^{\circ}$ and there are no significant differences between both materials. For irradiated samples, the water CA decreases, indicating that the materials become more hydrophilic after irradiation. On the other hand, for glycerol, no significant change in CA values is observed after irradiation. Finally, an increase of the CA values of paraffin oil happens after irradiation. In Fig. S3 and Table S1 the results for the composites with different concentrations of EG are shown. We did not observe any effect of the concentration on the obtained results.

As mentioned, the CA is related with the surface free energy components between the liquid $(l)$ and the surface of the solid substrate $(s)$. The model by Owens, Wendt, Rabel and Kaelble (OWRK-model) was applied in order to calculate the solid surface free energy components [12]. This model uses the Young's equation to determine the surface total energy $\gamma_{s}{ }^{T O T}$ and the dispersive $\gamma_{s}{ }^{d}$ and polar $\gamma_{s}{ }^{p}$ components of the surface tension. The surface free energy of the components of the liquid probes can be found in [46] and the calculated values are summarized in Table 2. Addition-

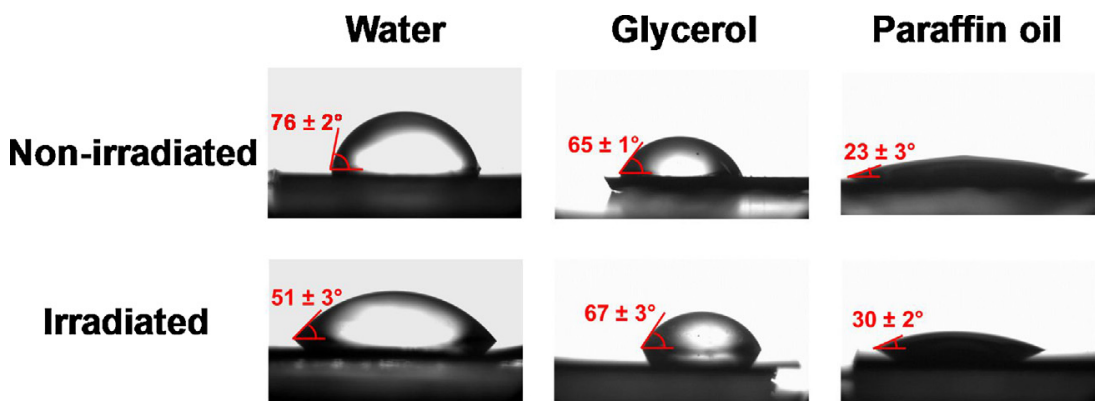

Fig. 5. Images of a drop of different liquids on the surface of non-irradiated and irradiated PET/EG 0.4 wt.\%. In all the images average CA values are indicated. 
Table 2

Surface free energy components $\left(\mathrm{mJ} / \mathrm{m}^{2}\right)$ of the different non-irradiated and irradiated samples using the OWRK-model: dispersive $\left(\gamma_{s}^{d}\right)$, polar $\left(\gamma_{s}^{p}\right)$ components and surface total energy $\left(\gamma_{s}{ }^{\text {TOT }}\right)$. Errors are estimated in ca. $10 \%$.

\begin{tabular}{llll}
\hline Sample & $\gamma_{S}^{d}$ & $\gamma_{S}^{p}$ & $\gamma_{S}^{\text {TOT }}$ \\
\hline PET, non-irradiated & 27.2 & 8.0 & 35.2 \\
PET, irradiated & 21.4 & 22.4 & 43.8 \\
PET/EG 0.4 wt.\%, non-irradiated & 26.3 & 8.6 & 34.9 \\
PET/EG 0.4 wt.\%, irradiated & 20.1 & 23.2 & 43.3 \\
\hline
\end{tabular}

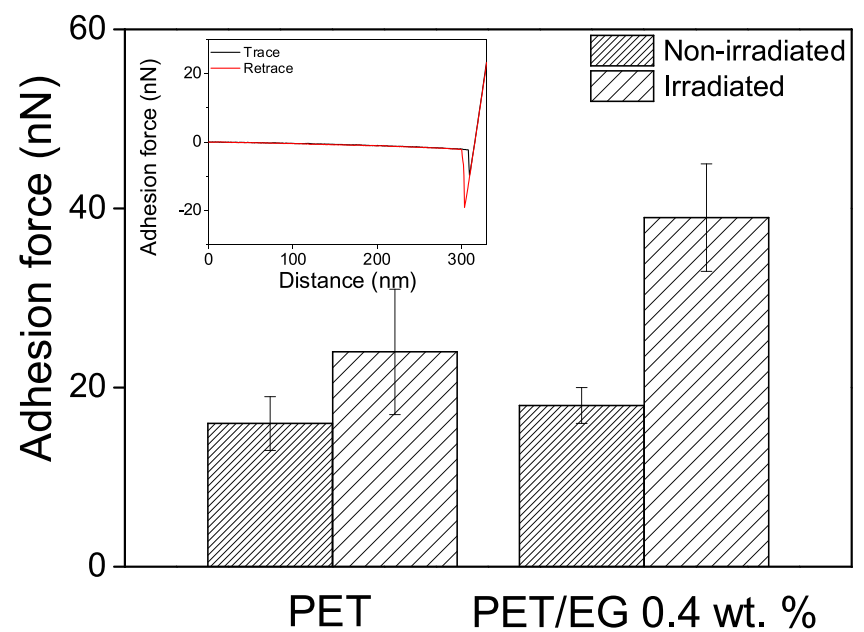

Fig. 6. Adhesion force values obtained by means of the colloidal probe technique, for non-irradiated and irradiated PET and PET/EG 0.4 wt.\% samples. The inset shows the force curves corresponding to the PET/EG 0.4 wt.\% sample.

ally, Table S2 shows the values obtained for the PET with different concentrations of EG.

According to the values obtained, there are no significant differences between PET and the nanocomposite. Comparing the results obtained for the non-irradiated and the irradiated samples, the dispersive component remains constant or slightly lower, while the polar component of the surface tension increases. The surface total energy also increases after irradiation.

Measurements of adhesion forces were performed using AFM and a colloidal tip. The inset in Fig. 6 shows the force curves corresponding to the PET/EG $0.4 \mathrm{wt}$ \% sample. From the retracting curve, the adhesion force value can be obtained, which corresponds to the difference between the baseline and the minimum force. The results of the analysis are summarized in Fig. 6. Before irradiation, the adhesion force for both the raw polymer and the composite has a similar value while it increases for both materials upon irradiation.

\section{Discussion}

In general, the mechanism of LIPSS formation by ns pulsed laser irradiation involves a thermal process in which a minimum value of fluence is needed to ensure that the surface is heated above $T_{g}$ or $T_{m}$ for amorphous or semicrystalline polymers respectively. This effect provides enough mobility to the polymer chains to rearrange. After heating by a single pulse, the dynamics of the LIPSS formation proceeds by a feedback mechanism provoked by repetitive irradiation. It has been previously reported that LIPSS can be formed in PET upon irradiation with ns pulses at $266 \mathrm{~nm}$ [36]. At this wavelength, the polymer absorbs efficiently and the surface is heated above its $\mathrm{T}_{\mathrm{g}}$, which is $72{ }^{\circ} \mathrm{C}$ for PET, as determined by differential scanning calorimetry (DSC). Moreover, in the case of PET/EG $0.4 \mathrm{wt} . \%$ the $\mathrm{T}_{\mathrm{g}}$ is $75^{\circ} \mathrm{C}$ and its melting temperature $\mathrm{T}_{\mathrm{m}}$ also plays a role, since it has been shown that the nanocomposite exhibits sig-

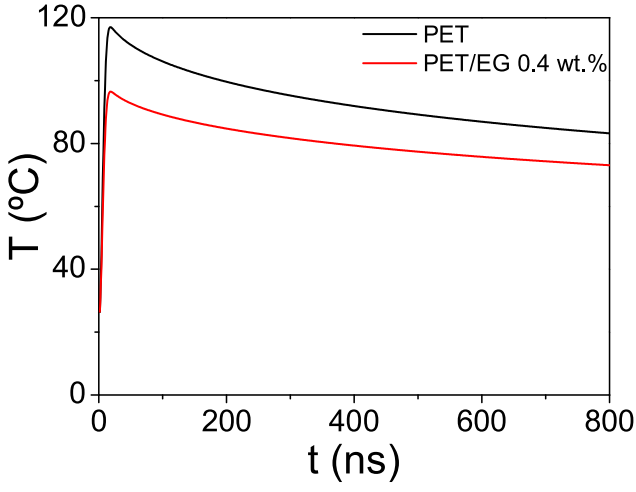

Fig. 7. Time dependence of the surface temperature for irradiation of PET and PET $/ 0.4 \mathrm{wt} . \%$ at $266 \mathrm{~nm}$ with a fluence of $8 \mathrm{~mJ} / \mathrm{cm}^{2}$.

nificant traces of crystallinity. In this case, $\mathrm{T}_{\mathrm{m}}$ has a value of $257^{\circ} \mathrm{C}$ as determined by differential scanning calorimetry. The intrinsic semicrystalline nature of the PET/EG nanocomposites implies that they must be heated above the melting temperature in order to reach enough mobility to reorganize. Accordingly, a larger number of pulses is needed in order to form LIPSS on PET/EG 0.4 wt.\% (ca. 1200 pulses) in comparison to raw PET (ca. 200 pulses). Additionally, the range of fluences at which LIPSS are formed is wider for the PET/EG nanocomposites. A further explanation needs to bear in mind two issues: the previously mentioned crystallinity of PET/EG samples (Figs. 3 and 4), and the different thermal properties as a consequence of the presence of the carbon nanoadditive.

The temperature reached upon irradiation with every single pulse can be estimated by solving the one-dimensional heat conduction equation following the approach reported previously $[36,47]$. For the sake of simplification, neither the temperature dependence of the polymer physical parameters nor the possible changes in the thermal properties of the surface caused by the previous laser pulses were taken into account. An increase in the thermal conductivity and in the heat capacity on similar composites of graphite/polymer has been already reported [48]. We consider that similar changes take place in our nanocomposites. The magnitudes used for calculations are: specific heat $1172 \mathrm{~J} / \mathrm{Kg} \mathrm{K}$ for PET and $1346 \mathrm{~J} / \mathrm{Kg} \mathrm{K}$ for PET/EG $0.4 \mathrm{wt} . \%$, thermal conductivity $0.15 \mathrm{~W} / \mathrm{m} \mathrm{K}$ for PET and $0.16 \mathrm{~W} / \mathrm{m} \mathrm{K}$ for PET/EG $0.4 \mathrm{wt} . \%$, and density $1390 \mathrm{Kg} / \mathrm{m}^{3}$ for PET and $1398 \mathrm{Kg} / \mathrm{m}^{3}$ for PET/EG $0.4 \mathrm{wt} . \%$ [49]. We obtain that the temperature reached at the surface decreases for the polymer containing EG as shown in Fig. 7. This could explain that the number of pulses needed for LIPSS formation in the case of PET/EG samples is larger in comparison to raw PET (Fig. 2a). Additionally, the wider range of fluences for LIPSS formation in the case of PET/EG is also in agreement with a higher thermal conductivity and thermal diffusivity (Fig. 2b).

On the other hand, the filler concentration clearly affects the LIPSS formation process (Fig. 3). The thermal conductivity and diffusivity increases as the filler increases, and thus the superficial temperature decreases because of a larger heat diffusion. A lower surface temperature will lead to a higher superficial viscosity, making the arrangement of material more difficult. Also, a higher thermal conductivity will induce a faster cooling of the material and the feedback effect involved in LIPSS formation will be less efficient [39].

The obtained results indicate that structural and thermal properties are determining for the LIPSS formation. Thus, it would be expected that the use of nanofillers, which can act as nucleant agents, will induce the formation of LIPSS upon irradiation with a larger number of pulses while the use of fillers, whose thermal properties are significantly different than the polymer matrix, will also affect the feedback mechanism involved in LIPSS formation. 
Chemical modifications might be expected upon laser irradiation, and in fact they have been reported previously [50]. However, this issue cannot be readily assessed in our experiments. This could be explained taking into account that the height of the nanostructures is below $100 \mathrm{~nm}$ while the penetration depth of the Raman excitation is around $700 \mathrm{~nm}$. It is documented that the bands detected in $1095 \mathrm{~cm}^{-1}$ and $1117 \mathrm{~cm}^{-1}$ are sensitive to the crystallinity of PET samples [45]. In one hand, the shoulder with a peak at $1117 \mathrm{~cm}^{-1}$ indicates the amorphous character of the polymer films. The rising of the Raman bands in $1095 \mathrm{~cm}^{-1}$ and $1117 \mathrm{~cm}^{-1}$ determines an increase in the crystalline content in the polymer matrix of the nanocomposite. This is also supported by the results shown in Fig. 3.

The CAs and surface energies obtained for the PET samples are in agreement with those reported in literature [50]. For the nanocomposites, the wettability of the nanocomposites is similar to that of the polymer surfaces indicating that, for the concentrations used, the nanoadditive is not changing the wettability. The surface hydrophilicity is observed to increase when the ripple-like nanostructures are induced in both materials (Table 1). Both the neat polymer and the composite exhibit similar increase after irradiation. This could be explained by considering on the one hand, the Wenzel model [51], which describes a homogeneous wetting regime and predicts that roughness enhances wettability and that in the case of a hydrophilic solid, it becomes more hydrophilic when rough. On the other hand, we have to take into account a slight oxidation of the polymer surface by irradiation in air [50]. Regarding the surface energy components, the increase of the polar component would also be in agreement with new functional groups at the surface of the polymer film and contribute to a decrease of the total surface energy value in both materials upon irradiation. The colloidal probe technique also gives information about the changes produced upon irradiation in the outmost layer of the surfaces. The differences observed follow the same trend, i.e. increase of the adhesion force in the case of irradiated samples, as the one obtained from the CA measurements. It has been previously reported that a decrease of the adhesion force occurs for nanostructured surfaces as a consequence of the decrease in the effective contact area $[52,53]$. However, in this case, this decrease seems to be compensated by the enhanced adhesion expected from the higher surface polarity. Thus, surface nanostructuring of the polymer and polymer composite takes place together with an increase of hydrophilicity, surface energy and surface adhesion, which may be relevant for the development of applications. In particular, in applications of polymer nanocomposites already reported as cell culture [54], the presence of nanostructures with increased hydrophilicity, surface energy and adhesion can promote both cell proliferation and differentiation. In the case of electronic devices and sensors, the presence of nanostructures may also improve their performance, and the increased surface energy and adhesion force will facilitate further functionalization of the surface and the fabrication of multilayer-systems towards all-organic devices.

\section{Conclusions}

Laser induced periodic surface structures were obtained in PET and PET reinforced with EG. The experimental parameters needed for LIPSS formation, in terms of number of pulses and fluence, are influenced by the presence of the nanoadditive. In particular, a higher number of pulses is needed in order to obtain periodic nanostructures in the case of the reinforced polymer. On the one hand, the crystallinity of the nanocomposites plays a role since the crystalline phase constitutes a robust scaffold providing mechanical stability to the material, and higher temperatures need to be reached in order to overcome the melting temperature so that superficial crystallites melt providing enough polymer dynamics. On the other hand, higher thermal conductivity and thermal diffusivity of these materials make the feedback mechanism less efficient since the temperature reached will be lower in the case of the nanocomposite. Additionally, at the same irradiation conditions, the LIPSS obtained on PET with a higher content of EG are smaller, also as a consequence of the higher thermal conductivity and diffusivity. Although significant chemical changes between non-irradiated and irradiated samples are not observed by Raman spectroscopy, contact angle measurements indicate that the materials become more hydrophilic and the surface free energy and its polar component increase after irradiation. This could be related to wetting in the Wenzel state due to the increase of roughness, together with chemical modifications on the material surface upon laser irradiation. Finally, adhesion force increases in the case of the nanostructured films.

\section{Acknowledgements}

The authors gratefully acknowledge financial support from the MINECO (FIS2013-44174-P, MAT2015-66443-C02-1-R, FIS201571933-REDT, CTQ2016-75880-P) and Junta de Castilla y León (Project SA046U16). RIR is grateful for the International Scholarship number 314197 provided by Consejo Nacional de Ciencia y Tecnología (CONACyT-México) and E.R. thanks MINECO for a Ramón y Cajal contract (RYC-2011-08069). The authors thank J. V. GarcíaRamos for the Raman measurements.

\section{Appendix A. Supplementary data}

Supplementary material related to this article can be found, in the online version, at doi:https://doi.org/10.1016/j.apsusc.2017.12. 147

\section{References}

[1] R. Sengupta, M. Bhattacharya, S. Bandyopadhyay, A.K. Bhowmick, A review on the mechanical and electrical properties of graphite and modified graphite reinforced polymer composites, Prog. Polym. Sci. 36 (2011) 638-670.

[2] K. Kalaitzidou, H. Fukushima, L.T. Drzal, Multifunctional polypropylene composites produced by incorporation of exfoliated graphite nanoplatelets, Carbon 45 (2007) 1446-1452.

[3] S. Stankovich, D.A. Dikin, G.H.B. Dommett, K.M. Kohlhaas, E.J. Zimney, E.A Stach, R.D. Piner, S.T. Nguyen, R.S. Ruoff, Graphene-based composite materials, Nature 442 (2006) 282-286.

[4] Z. Xu, C. Gao, In situ polymerization approach to graphene-reinforced nylon-6 composites, Macromolecules 43 (2010) 6716-6723.

[5] P.-C. Ma, N.A. Siddiqui, G. Marom, J.-K. Kim, Dispersion and functionalization of carbon nanotubes for polymer-based nanocomposites: a review, Compos. Part A: Appl. Sci. Manuf. 41 (2010) 1345-1367.

[6] J.N. Coleman, U. Khan, Y.K. Gun'ko, Mechanical reinforcement of polymers using carbon nanotubes, Adv. Mater. 18 (2006) 689-706.

[7] A. Ammar, A.M. Al-Enizi, M.A. AlMaadeed, A. Karim, Influence of graphene oxide on mechanical, morphological, barrier, and electrical properties of polymer membranes, Arab. J. Chem. 9 (2016) 274-286.

[8] S.H. Park, P.R. Bandaru, Improved mechanical properties of carbon nanotube/polymer composites through the use of carboxyl-epoxide functional group linkages, Polymer 51 (2010) 5071-5077.

[9] A. Linares, J.C. Canalda, M.E. Cagiao, M.C. García-Gutiérrez, A. Nogales, I. Martín-Gullón, J. Vera, T.A. Ezquerra, Broad-band electrical conductivity of high density polyethylene nanocomposites with carbon nanoadditives: multiwall carbon nanotubes and carbon nanofibers, Macromolecules 41 (2008) 7090-7097

[10] Z. Tao, H. Wang, X. Li, Z. Liu, Q. Guo, Expanded graphite/polydimethylsiloxane composites with high thermal conductivity, J. Appl. Polym. Sci. 134 (2017) 44843

[11] Z. Han, A. Fina, Thermal conductivity of carbon nanotubes and their polymer nanocomposites: a review, Prog. Polym. Sci. 36 (2011) 914-944.

[12] D.K. Owens, R.C. Wendt, Estimation of the surface free energy of polymers, J Appl. Polym. Sci. 13 (1969) 1741-1747

[13] A. Celzard, J.F. Marêché, G. Furdin, Modelling of exfoliated graphite, Prog. Mater. Sci. 50 (2005) 93-179.

[14] J.R. Potts, D.R. Dreyer, C.W. Bielawski, R.S. Ruoff, Graphene-based polymer nanocomposites, Polymer 52 (2011) 5-25. 
[15] R.K. Layek, A.K. Nandi, A review on synthesis and properties of polymer functionalized graphene, Polymer 54 (2013) 5087-5103.

[16] W. Zheng, S.-C. Wong, Electrical conductivity and dielectric properties of PMMA/expanded graphite composites, Compos. Sci. Technol. 63 (2003) $225-235$.

[17] R.K. Goyal, P.A. Jagadale, U.P. Mulik, Thermal, mechanical, and dielectric properties of polystyrene/expanded graphite nanocomposites, J. Appl. Polym. Sci. 111 (2009) 2071-2077.

[18] M. Zhang, D.-J. Li, D.-F. Wu, C.-H. Yan, P. Lu, G.-M. Qiu, Poly(ethylene terephthalate)/expanded graphite conductive composites: structure, properties, and transport behavior, J. Appl. Polym. Sci. 108 (2008) 1482-1489.

[19] S. Paszkiewicz, I. Pawelec, A. Szymczyk, Z. Špitalský, J. Mosnáček, A. Kochmańska, Z. Rosłaniec, Effect of exfoliated graphite nanoplatelets' size on the phase structure, electrical, and barrier properties of poly(trimethylene terephthalate)-based nanocomposites, Polym. Eng. Sci. 55 (2015) 2222-2230.

[20] X. Li, X. Yu, Y. Han, Polymer thin films for antireflection coatings, J. Mater. Chem. C 1 (2013) 2266-2285.

[21] S. Kim, U.T. Jung, S.-K. Kim, J.-H. Lee, H.S. Choi, C.-S. Kim, M.Y. Jeong, Nanostructured multifunctional surface with antireflective and antimicrobia characteristics, ACS Appl. Mater. Interfaces 7 (2015) 326-331.

[22] J.R.C. Smirnov, M. Ito, M.E. Calvo, C. López-López, A. Jiménez-Solano, J.F. Galisteo-López, P. Zavala-Rivera, K. Tanaka, E. Sivaniah, H. Míguez, Adaptable ultraviolet reflecting polymeric multilayer coatings of high refractive index contrast, Adv. Opt. Mater. 3 (2015) 1633-1639.

[23] C. Guo, S. Wang, H. Liu, L. Feng, Y. Song, L. Jiang, Wettability alteration of polymer surfaces produced by scraping, J. Adhes. Sci. Technol. 22 (2008) 395-402.

[24] S.-I. Na, S.-S. Kim, J. Jo, S.-H. Oh, J. Kim, D.-Y. Kim, Efficient polymer solar cells with surface relief gratings fabricated by simple soft lithography, Adv. Funct. Mater. 18 (2008) 3956-3963.

[25] J.J. Hernández, M.A. Monclús, I. Navarro-Baena, F. Viela, J.M. Molina-Aldareguia, I. Rodríguez, Multifunctional nano-engineered polymer surfaces with enhanced mechanical resistance and superhydrophobicity, Sci. Rep. 7 (2017), 43450.

[26] H. Schift, Nanoimprint lithography: an old story in modern times? A review, J. Vac. Sci. Technol. B: Microelectron. Nanometer Struct. Process. Measure. Phenom. 26 (2008) 458-480.

[27] D. Qin, Y. Xia, G.M. Whitesides, Soft lithography for micro- and nanoscale patterning, Nat. Protoc. 5 (2010) 491-502.

[28] L.J. Guo, Nanoimprint lithography: methods and material requirements, Adv. Mater. 19 (2007) 495-513.

[29] M. Sanz, E. Rebollar, R.A. Ganeev, M. Castillejo, Nanosecond laser-induced periodic surface structures on wide band-gap semiconductors, Appl. Surf. Sci. 278 (2013) 325-329.

[30] J. Bonse, S. Höhm, S.V. Kirner, A. Rosenfeld, J. Krüger, Laser-induced periodic surface structures-a scientific evergreen, IEEE J. Sel. Top. Quantum Electron. 23 (2017) 1-15

[31] P. Gregorčič, M. Sedlaček, B. Podgornik, J. Reif, Formation of laser-induced periodic surface structures (LIPSS) on tool steel by multiple picosecond laser pulses of different polarizations, Appl. Surf. Sci. 387 (2016) 698-706.

[32] A. Talbi, A. Petit, A. Melhem, A. Stolz, C. Boulmer-Leborgne, G. Gautier, T. Defforge, N. Semmar, Nanoparticles based laser-induced surface structures formation on mesoporous silicon by picosecond laser beam interaction, Appl. Surf. Sci. 374 (2016) 31-35.

[33] E. Rebollar, M. Castillejo, T.A. Ezquerra, Laser induced periodic surface structures on polymer films: from fundamentals to applications, Eur. Polym. J. 73 (2015) 162-174.

[34] S. Pérez, E. Rebollar, M. Oujja, M. Martín, M. Castillejo, Laser-induced periodic surface structuring of biopolymers, Appl. Phys. A 110 (2013) 683-690.

[35] P. Slepička, O. Neděla, N. Slepičková Kasálková, P. Sajdl, V. Švorčík, Periodic nanostructure induced on PEN surface by KrF laser irradiation, Int. J. Nanotechnol. 14 (2017) 399-409.
[36] E. Rebollar, S. Pérez, J.J. Hernández, I. Martín-Fabiani, D.R. Rueda, T.A. Ezquerra, M. Castillejo, Assessment and formation mechanism of laser-induced periodic surface structures on polymer spin-coated films in real and reciprocal space, Langmuir 27 (2011) 5596-5606.

[37] E. Rebollar, J.R. Vazquez de Aldana, J.A. Pérez-Hernández, T.A. Ezquerra, P. Moreno, M. Castillejo, Ultraviolet and infrared femtosecond laser induced periodic surface structures on thin polymer films, Appl. Phys. Lett. 100 (2012), 041106.

[38] M. Forster, W. Kautek, N. Faure, E. Audouard, R. Stoian, Periodic nanoscale structures on polyimide surfaces generated by temporally tailored femtosecond laser pulses, Phys. Chem. Chem. Phys. 13 (2011) 4155-4158.

[39] E. Rebollar, D.R. Rueda, I. Martín-Fabiani, Á. Rodríguez-Rodríguez, M.-C. García-Gutiérrez, G. Portale, M. Castillejo, T.A. Ezquerra, In situ monitoring of laser-induced periodic surface structures formation on polymer films by grazing incidence small-angle X-ray scattering, Langmuir 31 (2015) 3973-3981.

[40] D.W. Bäuerle, Laser Processing and Chemistry, Springer-Verlag, Berlin Heidelberg, 2011

[41] E. Rebollar, J.R. Vazquez de Aldana, I. Martin-Fabiani, M. Hernandez, D.R. Rueda, T.A. Ezquerra, C. Domingo, P. Moreno, M. Castillejo, Assessment of femtosecond laser induced periodic surface structures on polymer films, Phys. Chem. Chem. Phys. 15 (2013) 11287-11298.

[42] S. Paszkiewicz, A. Szymczyk, Z. Špitalský, M. Soccio, J. Mosnáček, T.A. Ezquerra, Z. Rosłaniec, Electrical conductivity of poly(ethylene terephthalate)/expanded graphite nanocomposites prepared by in situ polymerization, J. Polym. Sci. B Polym. Phys. 50 (2012) 1645-1652.

[43] W.A. Ducker, T.J. Senden, R.M. Pashley, Direct measurement of colloidal forces using an atomic force microscope, Nature 353 (1991) 239-241.

[44] F.J. Boerio, S.K. Bahl, G.E. McGraw, Vibrational analysis of polyethylene terephthalate and its deuterated derivatives, J. Polym. Sci.: Polym. Phys. Ed. 14 (1976) 1029-1046.

[45] R. Kattumenu, C.H. Lee, V.N. Bliznyuk, S. Singamaneni, Micro-Raman spectroscopy of nanostructures, in: C.S.S.R. Kumar (Ed.), Raman Spectroscopy for Nanomaterials Characterization, 2012, pp. 417-444.

[46] C.J. van Oss, Interfacial Forces in Aqueous Media, Marcel Dekker, New York, 1994.

[47] M. Csete, Z. Bor, Laser-induced periodic surface structure formation on polyethylene-terephthalate, Appl. Surf. Sci. 133 (1998) 5-16.

[48] S. Paszkiewicz, A. Szymczyk, R. Pilawka, B. Przybyszewski, A. Czulak, Z RosŁaniec, Improved thermal conductivity of poly(trimethylene terephthalate-block-poly(tetramethylene oxide) based nanocomposites containing hybrid single-walled carbon nanotubes/graphene nanoplatelets fillers, Adv. Polym. Tech. 36 (2017) 236-242.

[49] J.E. Mark, Physical Properties of Polymers Handbook, Springer-Verlag, New York, 2007.

[50] E. Rebollar, S. Perez, M. Hernandez, C. Domingo, M. Martin, T.A. Ezquerra, J.P. Garcia-Ruiz, M. Castillejo, Physicochemical modifications accompanying UV laser induced surface structures on poly(ethylene terephthalate) and their effect on adhesion of mesenchymal cells, Phys. Chem. Chem. Phys. 16 (2014) 17551-17559.

[51] R.N. Wenzel, Resistance of solid surfaces to wetting by water, Ind. Eng. Chem. 28 (1936) 988-994.

[52] Z. Burton, B. Bhushan, Hydrophobicity, adhesion, and friction properties of nanopatterned polymers and scale dependence for micro- and nanoelectromechanical systems, Nano Lett. 5 (2005) 1607-1613.

[53] E. Wohlfart, J.P. Fernández-Blázquez, E. Knoche, A. Bello, E. Pérez, E. Arzt, A. del Campo, Nanofibrillar patterns by plasma etching: the influence of polymer crystallinity and orientation in surface morphology, Macromolecules 43 (2010) 9908-9917.

[54] H. Fan, L. Wang, K. Zhao, N. Li, Z. Shi, Z. Ge, Z. Jin, Fabrication, mechanical properties, and biocompatibility of graphene-reinforced chitosan composites, Biomacromolecules 11 (2010) 2345-2351. 